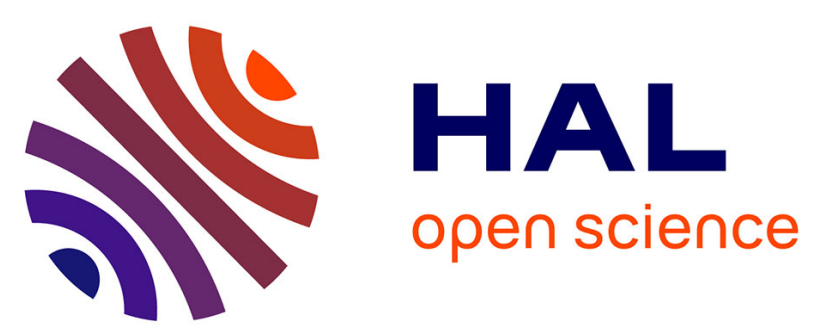

\title{
Conception and realization of a parallel-plate free-air ionization chamber for the absolute dosimetry of an ultrasoft X-ray beam
}

\author{
J.-E Groetz, Nabil Ounoughi, C Mavon, A Belafrites, M Fromm
}

\section{To cite this version:}

J.-E Groetz, Nabil Ounoughi, C Mavon, A Belafrites, M Fromm. Conception and realization of a parallel-plate free-air ionization chamber for the absolute dosimetry of an ultrasoft X-ray beam. Review of Scientific Instruments, 2014, 85, pp.83304. 10.1063/1.4890817 . hal-01117735

\author{
HAL Id: hal-01117735 \\ https://hal.science/hal-01117735
}

Submitted on 17 Feb 2015

HAL is a multi-disciplinary open access archive for the deposit and dissemination of scientific research documents, whether they are published or not. The documents may come from teaching and research institutions in France or abroad, or from public or private research centers.
L'archive ouverte pluridisciplinaire HAL, est destinée au dépôt et à la diffusion de documents scientifiques de niveau recherche, publiés ou non, émanant des établissements d'enseignement et de recherche français ou étrangers, des laboratoires publics ou privés. 


\title{
Conception and realization of a parallel-plate free-air ionization chamber for the
} absolute dosimetry of an ultrasoft $\mathrm{X}$-ray beam

\author{
J.-E. Groetz, ${ }^{1, a)}$ N. Ounoughi, ${ }^{1,2}$ C. Mavon, ${ }^{1}$ A.Belafrites, ${ }^{2}$ and M. Fromm ${ }^{1}$
}

1) Laboratoire Chrono-Environnement UMR CNRS 6249, Université de Franche-Comté, 16 route de Gray, 25030 Besançon Cedex, France

2) Laboratoire de Physique des Rayonnements et Applications, Université de Jijel, B.P. 98 Ouled Aissa, Jijel 18000, Algérie

(Dated: 20 June 2014)

We report the design of a millimeter-sized parallel plate free-air ionization chamber (IC) aimed at determining the absolute air kerma rate of an ultra-soft X-ray beam $(E=1.5 \mathrm{keV})$. The size of the IC was determined so that the measurement volume satisfies the condition of charged-particle equilibrium. The correction factors necessary to properly measure the absolute kerma using the IC have been established. Particular attention was given to the determination of the effective mean energy for the $1.5 \mathrm{keV}$ photons using the PENELOPE code. Other correction factors were determined by means of computer simulation (COMSOL and FLUKA). Measurements of air kerma rates under specific operating parameters of the lab-bench X-ray source have been performed at various distances from that source and compared to Monte-Carlo calculations. We show that the developed ionization chamber makes it possible to determine accurate photon fluence rates in routine work and will constitute substantial time-savings for future radiobiological experiments based on the use of ultra-soft X-rays.

a)jegroetz@univ-fcomte.fr 


\section{INTRODUCTION} insights into the radiobiological mechanisms that underline cellular inactivation and more generally cellular damage. ${ }^{1-6}$ In recent years, such low energy X-rays have also been employed to evaluate the efficacy of both USXs and secondary Low Energy Electrons (LEEs) at damaging $\mathrm{DNA}^{7,8}$. There are basically two main ways to use USX beams; access to a deliver $\mathrm{Al} \mathrm{K} \alpha$ photon beams $(1486.295 \mathrm{eV}, 1.5 \mathrm{keV}) .{ }^{15}$ Intensity of such USXs is dramatically reduced in air (i.e. a $4.8 \mathrm{~mm}$ thickness corresponds to $50 \%$ attenuation in standard conditions) and their dosimetry must therefore be particularly well-adapted. In order to properly characterize the generators dosimetry, an original millimeter-sized free-air ionizathe other hand, commercial Gafchromic dosimetric films (HD-810) were used in order to map the spatial dose distribution of the beam (not shown in details herein). ${ }^{15}$ Design of both the cold cathode USX generator and free-air ionization chamber were optimized by means of specific simulation tools: FLUKA $^{16,17}$ and PENELOPE ${ }^{18}$ calculation codes for simulation software environment for defining the geometry, meshing, solving and visualizing electric field lines in the devices. In this article, we report the main characteristics of the USX cold cathode generator, we describe the free-air ionization chamber design as well as its operation in terms of dosimetry, and we characterize the capabilities of the generator in terms of USX photons fluence rate delivery, notably for future radiobiological experiments. Prior to a full description of its geometry, we describe the way in which the dimensions of the chamber have first been optimized and validated. With a free air ionization chamber, if the ion-collecting gas volume is precisely known, the chamber can be considered as an absolute dosimeter. ${ }^{20,21}$ Nevertheless, the precise determination of the air kerma rate necessitates an 

tion factors where determined using both bibliographic references and computer simulations. Finally, kerma rate measurements make it possible to determine the photon fluence rate.

\section{MATERIALS AND METHODS}

The conception of the generator used in this work is based on a design first proposed of electron flow through ion impact on the cathode when a critical value of the electric field strength is reached, which depends on the gas density. Electrons interact with the atoms of the aluminum foil giving rise to the emission of characteristic $\mathrm{K} \alpha(1.5 \mathrm{keV})$ USXs accompanied by the emission of bremsstrahlung radiation. Depending on the parameters be suppressed or not: to produce a K $\alpha$ peak with our cold-cathode USX generator, the accelerating voltage must be set between 2 and $2.5 \mathrm{kV}{ }^{15}$

\section{A. Free-air ionization chamber}

Parallel-plate free air ionization chambers are widely used for the absolute measurement an ionization chamber. ${ }^{20-22,26}$ The photon beam passes through an aperture diaphragm, then between two parallel metal plates where an appropriate voltage $\left(V_{C}\right)$ is applied in order to collect the charges created by the interaction of the photons with the atomic components of air. The guard plates are used to ensure uniformity of the electric field lines in the volume

The air kerma rate for a free-air ionization chamber with a measuring volume $V_{m}$ is 


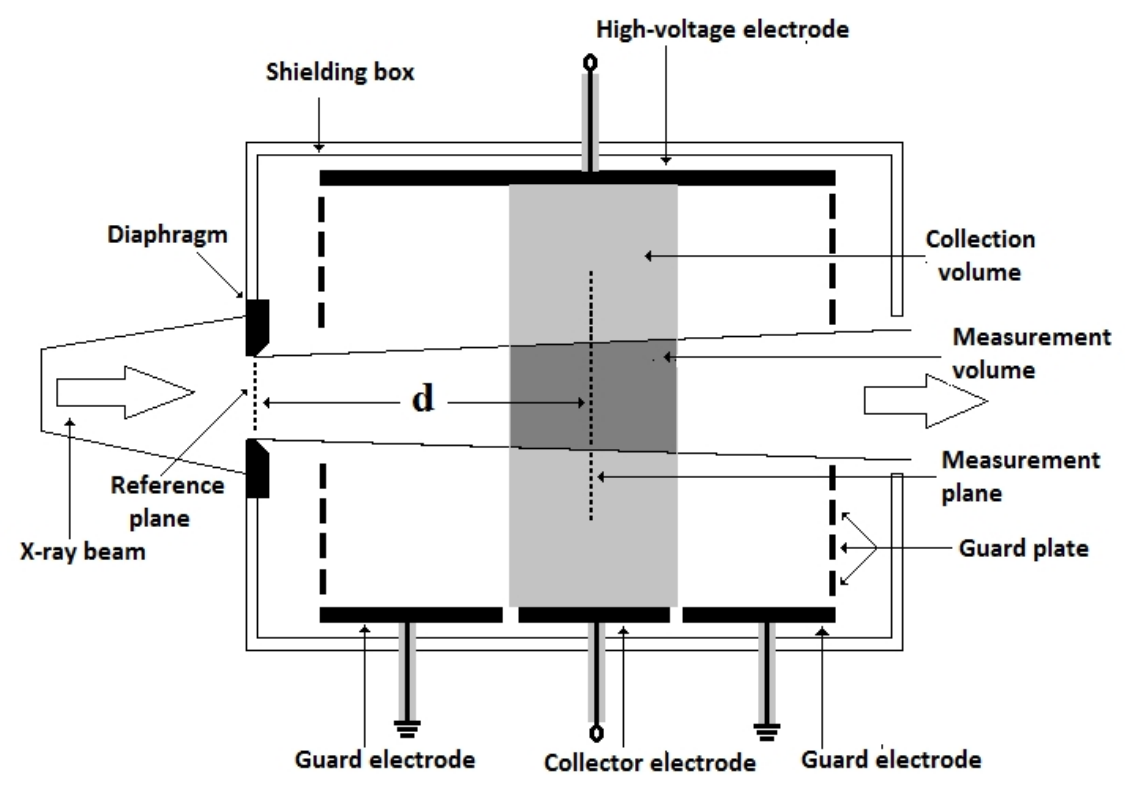

FIG. 1. Schematic diagram of a parallel-plate free-air ionization chamber, $d$ represents the distance between the reference plane and the measurement plane.

calculated by ${ }^{22}$ :

$$
\dot{K}=\frac{I}{\rho_{\text {air }} V_{m}} \times \frac{W_{\text {eff }}}{e} \times \frac{1}{1-g} \prod_{i} k_{i}
$$

where $I(\mathrm{~A})$ is the measured ionization chamber current, $\rho_{\text {air }}$ is the density of dry air under measured temperature and pressure conditions (at standard conditions $293.15 \mathrm{~K}$ and $101.33 \mathrm{hPa}, \rho_{\text {air }}=1.2048 \mathrm{~kg} \cdot \mathrm{m}^{-3}$ with relative uncertainty $0.01 \%$, as defined by NIST), $W_{\text {eff }}$ is the effective mean energy necessary to create an electron-ion pair in dry air ( $W_{\text {eff }}$ must be calculated for the $\mathrm{Al} \mathrm{K} \alpha$ X-rays, i.e. for $1.5 \mathrm{keV}$ photons, ${ }^{27,28}$ detailed in section III B) ; $e$ is the elementary charge; $g$ is the mean fraction of the secondary electron energy lost to bremsstrahlung radiation which is negligible when ultrasoft X-rays are absorbed in air ${ }^{21,29}$ and $\prod_{i} k_{i}$ is the dimensionless product of all necessary chamber correction factors. ${ }^{21,22,27,28,30-33}$

Some of these correction factors are straightforward to determine, while others must be assessed with specific calculations. In this work, the following correction factors have been used:

- $k_{\text {at }}$, the attenuation factor in air along the distance $d$ between reference and measurement plane ${ }^{22}$ (see Fig. 1.); since this factor depends on the density of air in the ambient 
conditions of temperature and pressure, it was calculated with these corrections; ${ }^{34}$

- $k_{\mathrm{TP}}$, the mass correction factor for taking account of the pressure and temperature variations. ${ }^{35}$ This factor has a low sensitivity to ambient pressure variations, but it is more sensitive to ambient temperature variations. In our case, an artificial weathering chamber is used to circulate dry or humid air in the irradiation chamber, to control both temperature and hygrometry;

- $k_{\mathrm{H}}$, the factor applied to correct the variation in the air hygrometry

- $k_{\mathrm{R}}$ is the factor for correcting the initial recombination in the collection volume, determined according to the two-voltage method; ${ }^{36,37}$ for small volume chambers, the general recombination is negligible; ${ }^{38} k_{\mathrm{R}}$ is determined based on a set of two electrical current measurements $I_{1}$ and $I_{2}$ at two different voltages $V_{1}$ and $V_{2}$ :

$k_{R}=1+\left(I_{1} / I_{2}-1\right) /\left(V_{1} / V_{2}-I_{1} / I_{2}\right)$

- $k_{p}$ is the polarization factor calculated using direct $\left(I_{+}\right)$and inverse $\left(I_{-}\right)$chamber currents $^{21}: k_{p}=\left(\left|I_{+}\right|+\left|I_{-}\right|\right) /\left(2\left|I_{-}\right|\right)$

- $k_{\text {Exc }}$ the factor to correct the excess charge due to the ion pairs produced by the initial photon interaction in air, ${ }^{22,27}$ calculated with the following relation $k_{\mathrm{Exc}}=1-1 / N_{\text {ion }}=$ $1-W(T) /\left(E \times \mu_{\mathrm{en}} / \mu\right)$; at $E=1500 \mathrm{eV}, \mu_{\mathrm{en}}$ and $\mu$ are very close $(0.25 \%$ difference $)$ and $\mathrm{W}(\mathrm{T})$ is equal to $34.36 \mathrm{eV}$ (see section III B);

- $k_{\text {Field }}$ the factor to correct the distortion of the electric field in the collection volume, ${ }^{39}$,

- $k_{e}$ and $k_{s}$ are respectively the correction factors for electron-loss and coherently scattered and fluorescence photons; ${ }^{30}$ those factors are generally determined with MonteCarlo simulations.

The values used in the present work and which have been easily calculated based on the relationships found in the literature are presented in Table 1. Uncertainties associated to the kerma rate measurements were calculated using the formula for propagation of uncertainties.

The factors $k_{\text {Field }}, k_{e}$ and $k_{s}$ have been computed using COMSOL ${ }^{\mathrm{TM}}$ and FLUKA calculation codes (see respectively section III.C. and section III.D). 
TABLE I. Corrections factors used in this work to calculate air kerma and which have been collected in the references listed above. $\mathrm{U}$ is the relative uncertainty in \%.

\begin{tabular}{llll}
\hline \hline \multicolumn{2}{l}{ Correction factor Value $\mathrm{U}(\%)$} & Specific conditions \\
\hline$k_{\mathrm{at}}$ & 2.498 & 1.44 & \\
$k_{\mathrm{TP}}$ & 1.024 & 0.68 & $300 \mathrm{~K}$ and $1013,25 \mathrm{hPa}$ \\
$k_{\mathrm{H}}$ & 0.998 & 0.10 & between $20 \%$ and $80 \%$ of hygrometry \\
$k_{\mathrm{R}}$ & 1.026 & 0.30 & \\
$k_{p}$ & 1.010 & 1.50 & \\
$k_{\mathrm{Exc}}$ & 0.977 & 0.12 & \\
\hline \hline
\end{tabular}

\section{B. Electric field simulations}

$\mathrm{COMSOL}^{\mathrm{TM}}$ has been used for optimizing the ionization chamber dimensions and operating parameters, using the $\mathrm{AC} / \mathrm{DC}$ module which provides an environment for the simulation of electrostatic phenomena. Parallel-plate free-air chambers must have a uniform electric field between their plates to ensure that the dimensions of the ion-collection volume and the length of the volume are accurately known.

\section{Particle physics Monte-Carlo simulations - FLUKA and PENELOPE codes}

FLUKA is a multipurpose Monte-Carlo simulation code, mainly dedicated to high energy physics and radiation dosimetry. ${ }^{16,17}$ Now, its use has been extended to nuclear medicine dosimetry and hadrontherapy, ${ }^{40,41}$ and it can be used to determine, with accuracy, the correction factors for the electron losses, photon scattering and photon fluorescence in a free-air ionization chamber. Energy cut-off for transport of primary electrons and photons is $1 \mathrm{keV}$, but this parameter can be lowered to $0.1 \mathrm{keV}$ for secondary photons. Fluorescence emission for $\mathrm{K}$ and $\mathrm{L}$ lines are well considered, and for charged particle transport such as electrons, a single scattering approach could be specified through the MULSOPT option. Since the aluminum foil (X-ray source) and the ionization chamber are close together, we must be aware of the beam divergence of our system. ${ }^{15}$ Beam divergence can be specified with the appropriate angular distribution.

PENELOPE is a Monte-Carlo code system for simulation of electron and photon 
transport. ${ }^{18}$ Both detailed simulations (hard events or analog histories) and multiple-

\section{FEATURES OF THE IONIZATION CHAMBER AND CORRECTION FACTOR CALCULATIONS}

\section{A. Design of the free-air ionization chamber} on the quality of the beam and the area of interest. Based on the schematic diagram of Fig. 1, a parallel-plate ionization chamber suitable for measuring the air kerma of USXs (typically $<5 \mathrm{keV}$ ) has been developed (Fig. 2). The use of such an ionization chamber requires that the measurement volume $\left(V_{m}\right)$ satisfies the condition of charged-particle equilibrium (i.e. the number of charged particles of each type, leaving a volume are equal to the number entering). ${ }^{20}$ This is ensured by the fact that the distance between the reference plane and the plane of measurement (see Fig. 1) is greater than the maximum range $R_{\text {air }}$ of the secondary electrons created by photons in air. From the CSDA, the value $R_{\text {air }}$ is estimated at $2.40 \mathrm{~mm}$ for $10 \mathrm{keV}$ photons (with ESTAR ${ }^{42}$ and PENELOPE) and at $0.15 \mathrm{~mm}$ for $2 \mathrm{keV}$ photons (with PENELOPE calculations, since ESTAR calculates the CSDA range down to $10 \mathrm{keV})$. For the secondary electrons created in the volume $V_{m}$, which dissipate their energy before being collected, it is a necessary condition that the distance from the latter to the electrodes remains greater than $R_{\text {air }}$. Two possible distances $d$ (corresponding to 6.5 and $10.5 \mathrm{~mm}$ starting from the reference plane) for the collector electrodes (see Fig. 2(a) and 2(b)) are available with our IC. For this application, only the electrodes at $6.5 \mathrm{~mm}$ were used, because the attenuation factor in air at $10.5 \mathrm{~mm}$ is too large, leading to values for the current too low to be measured. 


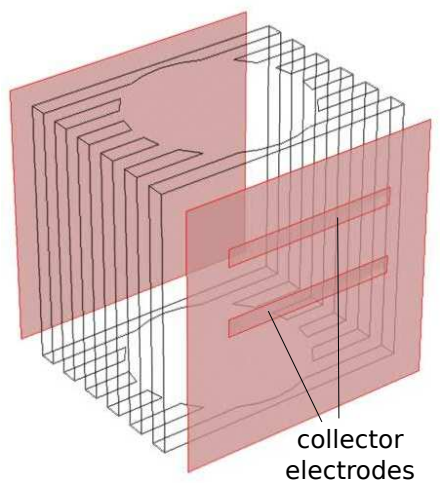

(a)

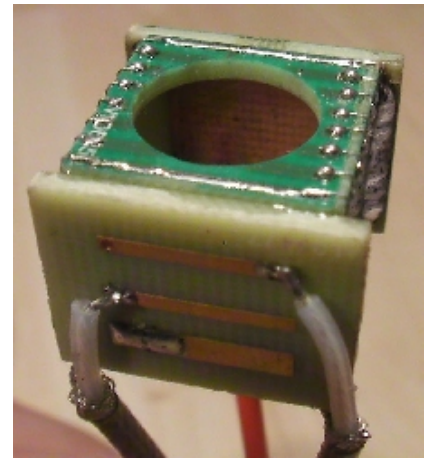

(b)

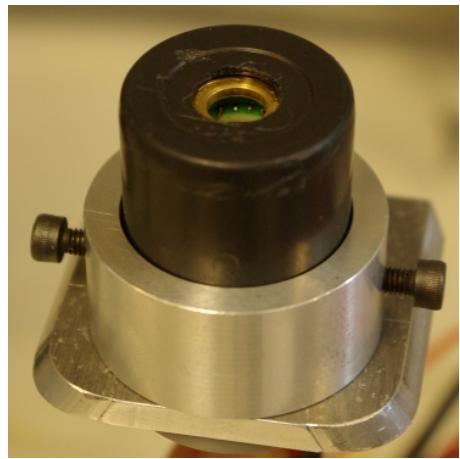

(c)

FIG. 2. Free-air ionization chamber: (a) 3D $\mathrm{COMSOL}^{\mathrm{TM}}$ representation of the chamber showing electrodes and guard plates (in black); (b) Picture of the design of the chamber without shielding box, where the welding points of the CMS resistances that connect consecutive guard plates are visible. Collector electrodes with connections are on the front side; (c) Picture of the final design with a cylindrical shielding box containing the copper diaphragm ( $8 \mathrm{~mm}$ diameter aperture) and electric insulation made up of black High-Density Poly-Ethylene (HDPE).

Both diaphragm and aperture (see Fig. 2) define the size of the X-ray beam entering the chamber. The mass attenuation coefficient for copper at $1.5 \mathrm{keV}$ (the energy of the $\mathrm{Al}$ $\mathrm{K} \alpha$ line) is $4.42 \times 10^{3} \mathrm{~cm}^{2} / \mathrm{g}$. The copper diaphragm machined for the ionizing chamber is actually $3 \mathrm{~mm}$ thick which ensures a complete attenuation of the considered photons. Copper is also used to machine the electrodes and guard plates, all maintained by polystyrene plates. Table II presents the sizes of the ionizing chamber. It should be noticed that this chamber is intended to measure air kerma rates of USXs (i.e. with energies less than $5 \mathrm{keV}$ ) and therefore, we can consider that $R_{\text {air }}<2.4 \mathrm{~mm}$. As for the diameter of the copper diaphragm aperture, we have chosen a $8 \mathrm{~mm}$ value due to the fact that we envisage the use of the X-ray source mainly to irradiate nanometer-scaled DNA layers ${ }^{43}$ in order to obtain data of radiobiological importance. Briefly, the DNA deposition method developed recently by our group $^{43}$ consists of a droplet of constant volume, being deposited on a hydrophobic graphite surface, which ensures a constant area (with diameter $=8 \mathrm{~mm}$ ) is created, this is why we selected the above mentioned area for the ionization chamber aperture. 
TABLE II. Free-air ionization chamber geometrical parameters.

\begin{tabular}{lc}
\hline \hline Chamber parameters & Dimensions $(\mathrm{mm})$ \\
\hline Aperture diameter of copper diaphragm & $8.00 \pm 0.02$ \\
Air path length $(d)$ & $6.50 \pm 0.02$ \\
Collecting length & $12.000 \pm 0.005$ \\
Electrode separation & $15.00 \pm 0.02$ \\
Collector width & $1.000 \pm 0.005$ \\
Guard plate width & $1.000 \pm 0.005$ \\
\hline Measuring volume $\left(\mathrm{mm}^{3}\right)$ & $52.78 \pm 0.36$ \\
\hline \hline
\end{tabular}

\section{B. Determination of $W_{\text {eff }}$}

The effective value of the energy expended in air per electron-ion pair must be calculated from the spectrum of secondary electrons generated, in our case, by $1.5 \mathrm{keV}$ photons. Within the chosen operating parameters, we can consider that the beam is monoenergetic. For photons of energy $E, W_{\text {eff }}$ is defined by the following relation: ${ }^{27}$

$$
W_{\mathrm{eff}}(E)=\frac{\int_{0}^{E_{\max }}\left(\frac{d \phi}{d t}\right) T d T}{\int_{0}^{E_{\max }}\left(\frac{d \phi}{d t}\right) \frac{T}{W(T)} d T}
$$

where $E_{\max }$ is the maximum energy transfered to electrons, $d \phi / d t$ is the spectral fluence of electrons liberated by photon interactions in air and $W(T)$ is the mean energy required to create an ion pair in air by an electron with a kinetic energy $T$.

Buhr et al. ${ }^{28}$ used a parameterization of the $\mathrm{W}$ value based on a fit to experimental values, which yields to:

$$
W(T)=\frac{W_{\text {air }}}{1-\left(T / A_{1}\right)^{A_{2}+A_{3} T}}
$$

with $A_{1}=14.636 \mathrm{eV}, A_{2}=-0.828$ and $A_{3}=-3.8 \times 10^{-5} \mathrm{eV}^{-1}$ (this last parameter is defined for electron energies $T>A_{1}$, meaning that no ion pairs are created by electrons with energies below $A_{1}$ ).

For this study, the PENELOPE-2011 ${ }^{18}$ code was used to determine the electron spectral fluence generated by the $1.5 \mathrm{keV}$ photons in air, which simulates photoionizations for K-shells and L-shells and relaxations including Auger electrons down to $100 \mathrm{eV}$. Figure 3 illustrates 
the spectral fluence of electrons from the $\mathrm{K} \alpha \mathrm{Al}$ X-rays calculated with PENELOPE-2011. These simulated results of $d \phi / d t$ and parameterization of $W(T)$ (Eq. (3)) were implemented in Eq. (2) to assess $W_{\text {air }}$. This leads to a value of $W_{\text {air }} / e$ for $1.5 \mathrm{keV}$ photons equal to $35.50 \pm 0.01 \mathrm{~J} / \mathrm{C}$. This value is in good accordance with the evolution of $W_{\text {eff }}$ as proposed in Bermann et al. ${ }^{27}$ In our case, the $W_{\text {eff }}$ is not corrected directly by the excess charge, as Buhr et al. proposed in their model. ${ }^{28}$ Therefore the excess charge factor $k_{\text {Exc }}$ was used for that correction.

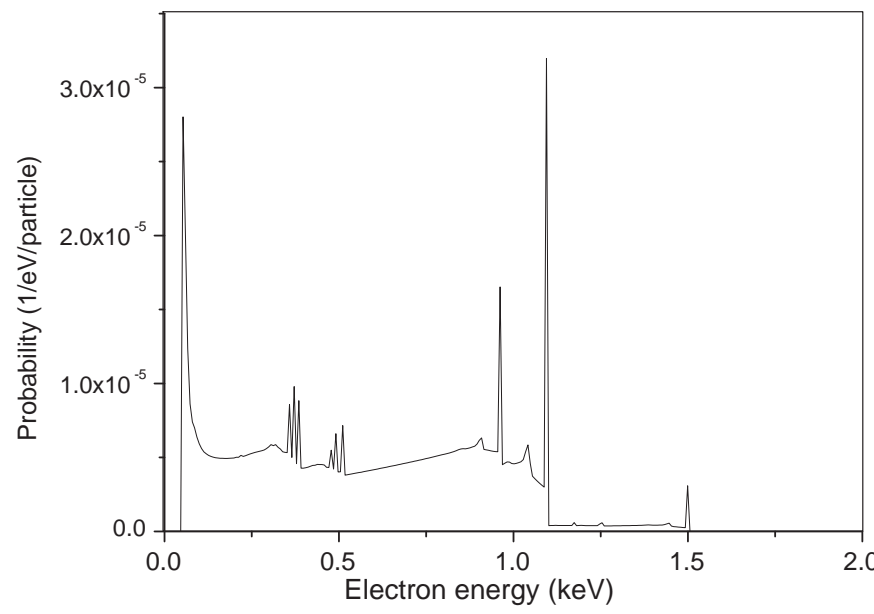

FIG. 3. Electron fluence spectrum from $1.5 \mathrm{keV}$ photons in air calculated with PENELOPE-2011

\section{Distribution of electric field lines in the chamber, correction factor $k_{\text {Field }}$}

Fig. 4 shows the distribution of electric field lines in the chamber as simulated using the COMSOL $^{\mathrm{TM}_{\mathrm{S}}}$ software; in the case of voltages applied to the shielding box equal to 0 and to half of the voltage applied to the chamber, $V_{C}$. These simulations clearly show how the shielding box voltage influences the field lines inside the ionization chamber. Indeed, when half of the $V_{C}$ is applied, the collection volume can be used nearer to the chamber aperture, which minimizes the distance $d$ and therefore minimizes the attenuation factor $k_{\text {at. }}$. When applying a voltage equal to half $V_{C}$ to the shielding box, we minimize the distortion of field lines in the volume of collection (see Fig. 4(b)), and we can thus use a large aperture (8 $\mathrm{mm}$ ). This will allow the penetration of a beam of large dimension in the chamber, thus generating larger signals that increase the sensitivity. ${ }^{33}$ The value of $V_{C} / 2$ will be used in further work and the correction factor $k_{\text {Field }}$ is determined using this particular voltage. 


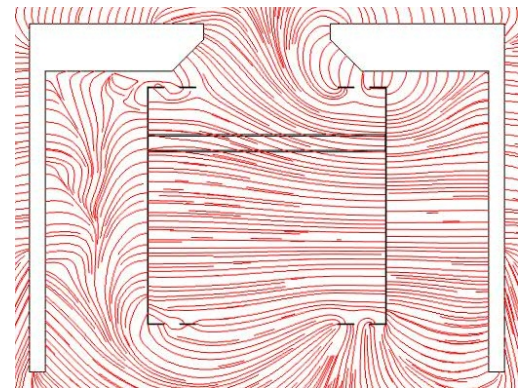

(a)

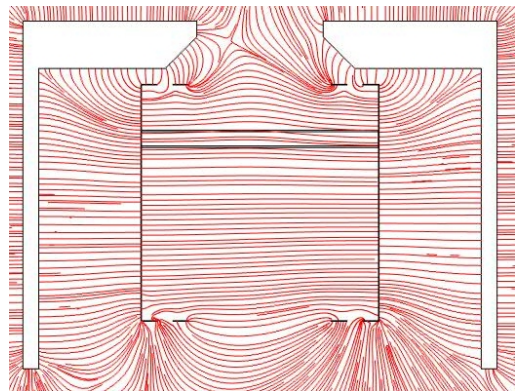

(b)

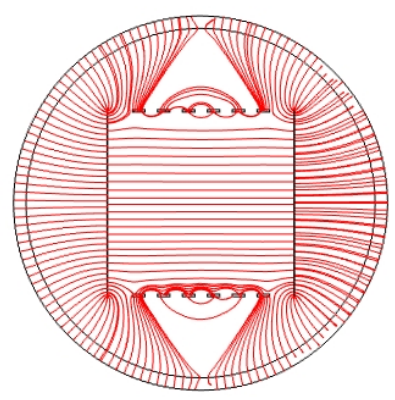

(c)

FIG. 4. Distribution of electric field lines in the ionization chamber. The black rectangle between electrodes represents the limits of the collection volume. (a) Field lines when the copper shielding box (in white color) is plugged to the ground; (b) Simulation corresponding to the case where the shielding box is polarized at $V_{C} / 2$. (c) Radial cross-sectional view of the field lines when the shielding box is at $V_{C} / 2$.

The determination of the correction factor $k_{\text {Field }}$ is based on the method described in detail in a report of the CIPM (International Committee for Weights and Measures) metrology portal. ${ }^{39}$ In brief, a digitized picture of the field lines in the collection volume of the ionization chamber (see for example Fig. 4(b)) is used to determine the parameters in Eq. (4). The field lines that pass through the center of the gap between the guards and collector are followed until they cross the central axis of the chamber. Using the intersection of this field line with the central axis, the offsets of the field lines along this axis $(x)$ are determined. Fig. 5 illustrates the method. The correction factor $k_{\text {Field }}$ is calculated using ${ }^{39}$ :

$$
k_{\text {Field }}=1+\frac{2 \Delta x}{L_{\mathrm{el}}+L_{\mathrm{g}}}
$$

Parameters $L_{\mathrm{el}}$ and $L_{\mathrm{g}}$ are implicit values depending on the design of the chamber; in the present study: $L_{\mathrm{el}}=1 \mathrm{~mm}$ and $L_{\mathrm{g}}=50 \mu \mathrm{m}$. The numerical values determined using the above-mentioned method are $\Delta x_{1}=0.06 \mathrm{~mm}$ and $\Delta x=0.04 \mathrm{~mm}$, leading to an average value of $0.05 \mathrm{~mm}$. The correction factor accounting for field effects in the collection volume of the chamber is thus $k_{\text {Field }}=1.095 \pm 0.10 \%$. 


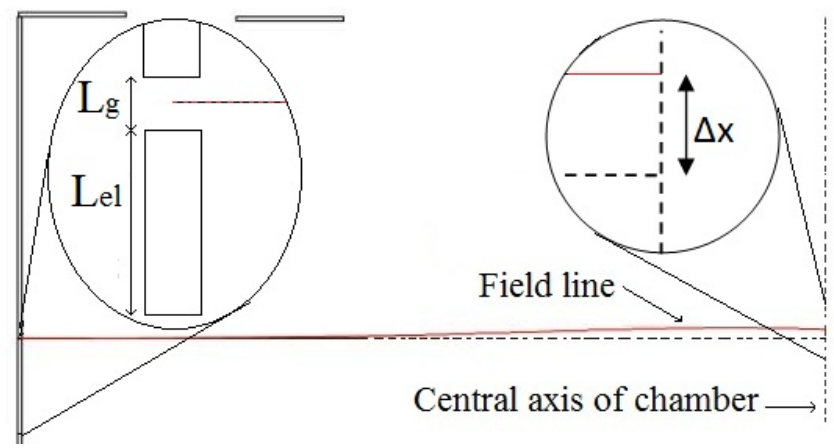

FIG. 5. Schematic representation of the parameters $\Delta x, L_{\mathrm{el}}$ and $L_{\mathrm{g}}$ used in Eq. (4) for the determination of $k_{\text {Field }}$.

\section{Correction factors $k_{e}$ and $k_{s}$}

In this part, the collection and detection volumes must be carefully described. The collection volume matches the volume of air inside the ionization chamber. As to the detection volume, it corresponds to the electrodes, the air space between the inner side and the collection volume (in the plane $\mathrm{YZ}$ ), the wall surrounding the collection volume and the collection volume itself.

Figure 6 shows the different regions used in FLUKA, corresponding to the different volumes:

- collection volume: region C31 (air)

- air space between inner side and collection volume: region C34 (upper) and region C35 (lower)

- IC wall : region C30 (epoxy)

- IC wall surrounding the collection volume : region C36 (epoxy)

- electrodes : upper region C32 and lower region C33 (copper)

- air (excluding the air regions above) : region $\mathrm{C} 10$

In our case, the detection volume is made up of regions C31, C32, C33, C34, C35 and C36, while the collection volume corresponds to region C31.

The correction factor $k_{e}$ is used to take into account the energy loss due to the primary electrons reaching the IC electrodes, the inner wall or the air space, and a partial energy 


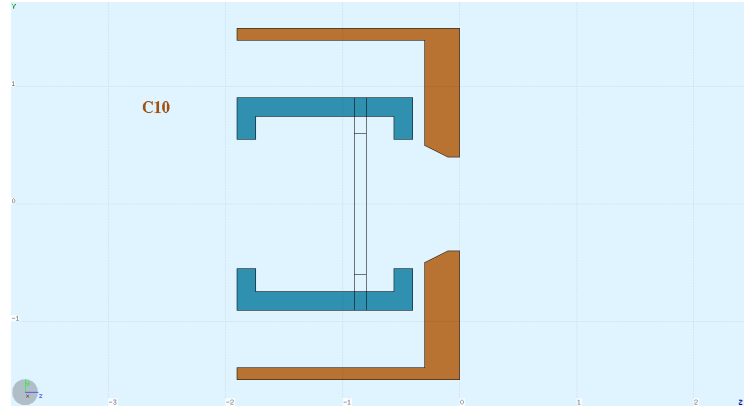

(a)

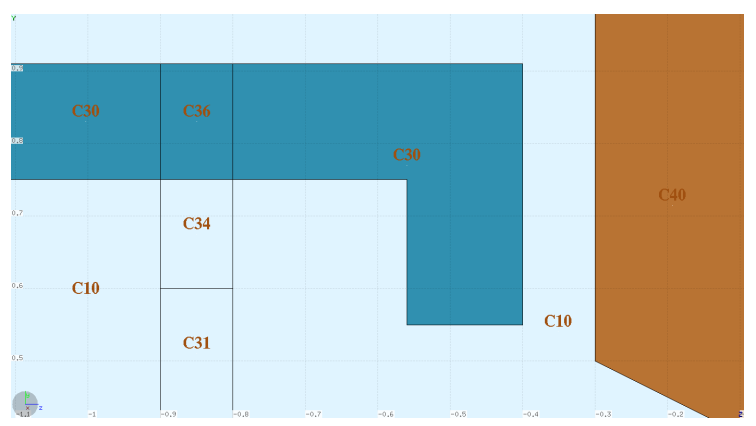

(c)

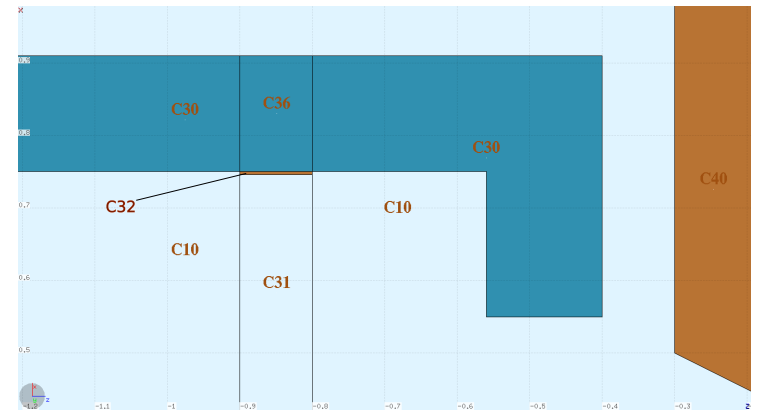

(b)

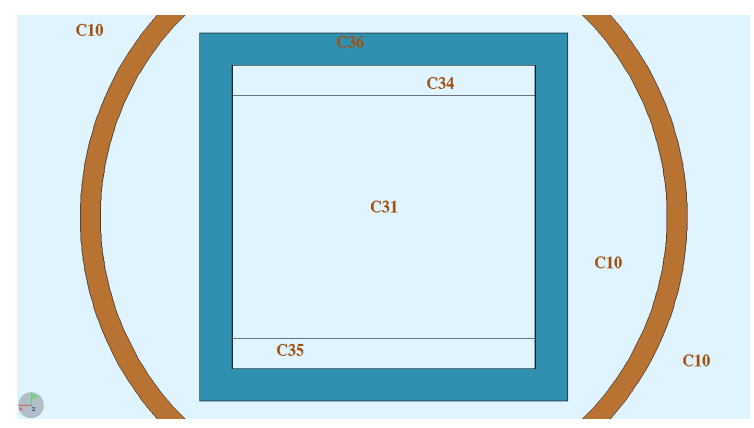

(d)

FIG. 6. 2D representation of the ionization chamber: (a) $2 \mathrm{D}$ section in ZX with the IC walls in blue and the shielding box in brown; 2D section with the different regions corresponding to the collection and detection volumes in ZX, also with the region C32 showing the upper electrode (b); in $\mathrm{ZY}(\mathrm{c})$ and in $\mathrm{XY}(\mathrm{d})$.

deposition in the collection volume. It can be defined $\mathrm{as}^{30}$ :

$$
k_{e}=\frac{E_{\mathrm{det}}}{E_{\mathrm{coll}}}=\frac{E_{\mathrm{elec}}+E_{\mathrm{vol}}+E_{\mathrm{coll}}}{E_{\mathrm{coll}}}
$$

where $E_{\text {det }}$ is the energy deposited in the detection volume, $E_{\text {elec }}$ is the energy deposited in the electrode plates (regions C32 and C33), $E_{\mathrm{vol}}$ is the energy deposited in the air space between the inner sides and the collection volume (regions C34 and C35) and $E_{\text {coll }}$ is the energy deposited in the collection volume by primary electrons.

The correction factor $k_{s}$ is defined for the scattered radiation producing additional electrons, which occurs when incident photons scatter on the diaphragm edge or when photons are produced by fluorescence. This factor $k_{s}$ is calculated $\operatorname{as}^{30}$

$$
k_{s}=\frac{E_{\mathrm{coll}}}{S_{\mathrm{coll}}}
$$


where $S_{\text {coll }}$ is the energy deposited by secondary electrons from scattered and fluorescence photons.

Both correction factors were calculated at various distances, corresponding to the range of use of the ionization chamber: 10, 12, 15, 20, 25 and $30 \mathrm{~mm}$ between the aluminum foil and the shielding box diaphragm (Fig. 7).

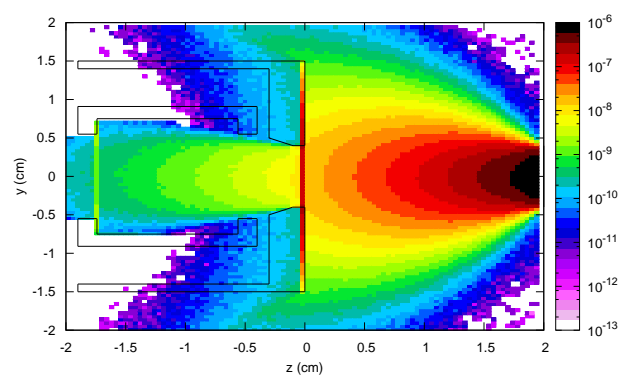

(a)

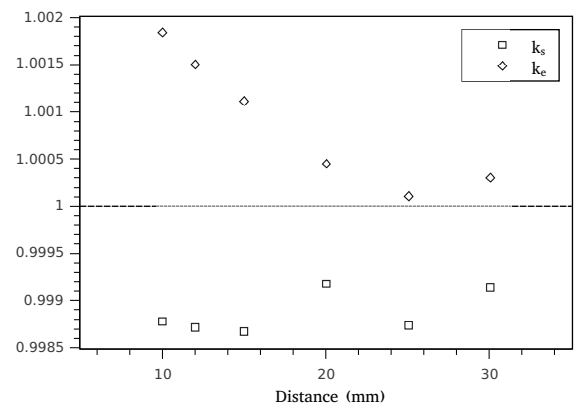

(b)

FIG. 7. (a) $2 \mathrm{D}$ representation of the deposed energy (normalized to $\mathrm{GeV} / \mathrm{cm}^{3} /$ primary) in the IC.

(b) Correction factors $k_{e}$ and $k_{s}$ as a function of the distance source-shielding box diaphragm.

From these results, we can deduce the average values and associated standard deviations $k_{e}=1.001 \pm 0.001$. and $k_{s}=0.999 \pm 0.001$.

\section{RESULTS AND DISCUSSION}

\section{A. Air kerma rate}

Aimed at determining the photon fluence rate $\dot{\varphi}$ in radiobiology experiments, the chamber provides air kerma rate $\dot{K}$ measurements. In case the distance between the USX source and the chamber must be varied, a step by step motor is used to move the chamber at the required distance. Air kerma rate $\dot{K}$ measurements were performed at various distances from the source (plane of the aluminum foil) of our lab-bench cold cathode generator in dry air $(2.4 \mathrm{kV}$ and $3 \mathrm{~mA})$. The dose is also calculated using FLUKA at the same distances, with special attention given to the angular distribution of the beam (Gaussian divergence at 0.7 rad). ${ }^{15}$ Concerning FLUKA calculations it should be noticed that the electronic equilibrium being established in the ionization chamber volume, absorbed dose in air and air kerma are identical. In Fig. 8, measurements and calculations are compared, where FLUKA results 
are expressed as air kerma per primary photons. Both results show a very good accordance with the distance source-ionization chamber.

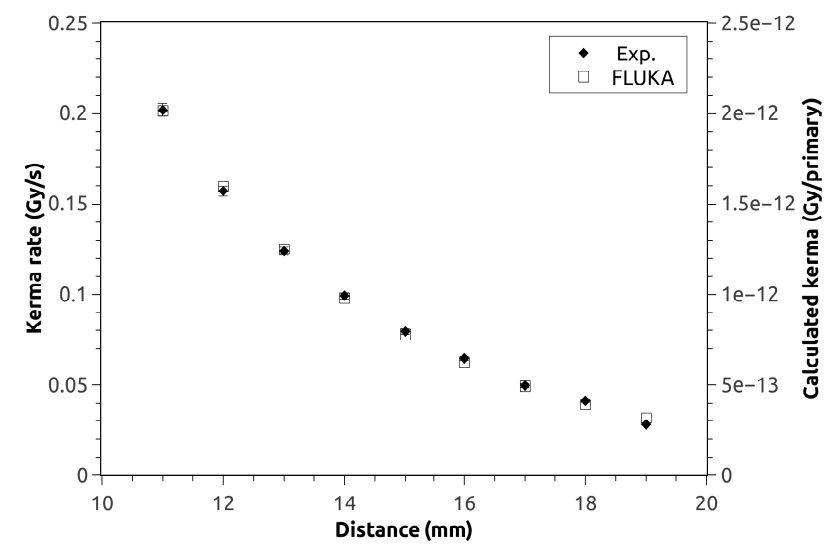

FIG. 8. Air kerma rate measurements with the ionization chamber at distances between 11 and $19 \mathrm{~mm}$ from the foil source (left axis), as compared to air kerma FLUKA calculation (right axis). Experimental errors are sometimes shorter than the black dots and can therefore not be seen on the plot.

\section{B. Photon fluence rate}

In the special case where the USX beam is of a mono-energetic character (under specific operating conditions, our cold-cathode USX generator acts as a mono-energetic source, see

Ref. 15 for more details), the measured kerma rate can be converted to a photon fluence rate using:

$$
\dot{\varphi}=\dot{K} \times\left[E \times\left(\frac{\mu_{\mathrm{en}}}{\rho}\right)_{\mathrm{air}}\right]^{-1}
$$

where $E$ is the mean photon energy and $\left(\mu_{\mathrm{en}} / \rho\right)$ air is the mass energy-absorption coefficient for $1.5 \mathrm{keV}$ X-rays in dry air, equal to $1.188 \times 10^{3} \mathrm{~cm}^{2} \cdot \mathrm{g}^{-1}$. At constant acceleration voltage, the measured photon fluence rate increases linearly with the generators current. ${ }^{15}$

We measured the photon flux through a detector Si (PIN)-type XR-100 CR Amptek ${ }^{\mathrm{TM}}$ with a sensitive area of $6.72 \mathrm{~mm}$ and a beryllium shielding foil having a thickness of 12.5 $\mu \mathrm{m}$ (the correction due to the attenuation in the beryllium foil was taken into account). Operating conditions for the USX generator were $2 \mathrm{kV}$ and $1.5 \mathrm{~mA}$, in order to minimize 
pulse pile-up and radiation damages to the detector, as well as taking maximum benefit of highest beam purity (96\%). Measurements were also performed for distances to the aluminum foil remaining beyond $50 \mathrm{~mm}$ for the same reasons.

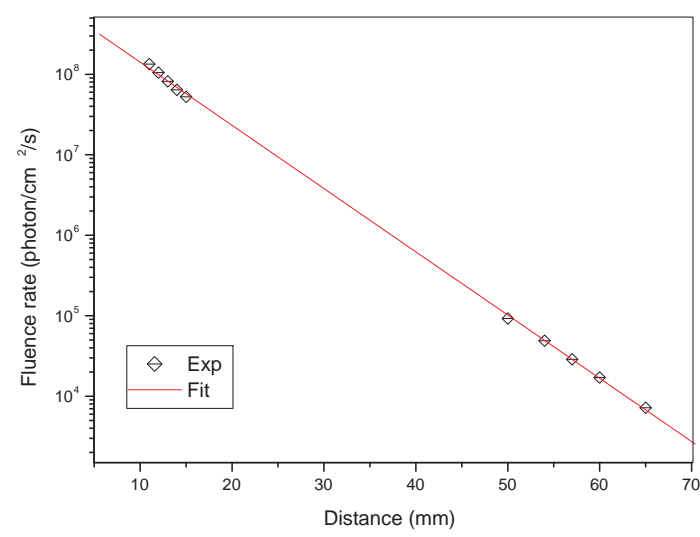

FIG. 9. Photon fluence rate measurements with the ionization chamber at distances between 11 and $15 \mathrm{~mm}$ from the foil source and with the Si diode from 50 to $65 \mathrm{~mm}$.

Fig. 9 presents the photon fluence rate measured with the IC and the Si diode in their range of distances (between 11 and $15 \mathrm{~mm}$ for the $\mathrm{IC}$, from 50 to $65 \mathrm{~mm}$ for the diode). The decreasing exponential fit shows a good correlation for both detectors. Nevertheless, it should be difficult to have a perfect correlation, due to the beam geometry and that of both measuring systems. Indeed the spatial fluence distribution of the beam is not homogeneous close to the aluminum foil (in the IC distance range of measurement), and becomes more homogeneous away from the source. ${ }^{15}$ Moreover, measurements carried out by the IC are averaged over an area of $50 \mathrm{~mm}^{2}$ (aperture area), while those performed using the Si (PIN) are averaged over an area of $6.75 \mathrm{~mm}^{2}$ (sensitive surface of the detector).

\section{CONCLUSION}

We have successfully designed an original millimeter-sized parallel plate free air ionization chamber specially intended to determine the photon fluence rates of a mono-energetic USXs beam delivered in air. It required a precise determination of the correction factors at a very low energy value (i.e. $1.5 \mathrm{keV}$ photon energy), especially for the effective mean energy $W_{\text {eff }}$, the field correction factor and the correction factors for electron loss and fluorescence photons in the collection volume. 
The measured absolute kerma rate in air compared to Monte-Carlo simulations have shown good agreement within the experimental uncertainties range. The evolution of fluence rates related to the distance from the source were also performed with the IC and a Si-diode and have shown a good correlation. Further work will consist in strictly validate the photon fluence rate with a suitable and calibrated diode.

The ionization chamber developed herein will allow considerable time savings in future radio-biological experiments, where DNA layers with nanometer-scaled thicknesses will be exposed to USXs in order to generate low energy photo-electrons $(0-10 \mathrm{eV})$ from a graphite substrate. Additionally, such an ionization chamber may provide useful in research areas where Ultra-Soft X-rays are used. Lastly, the work presented in this paper completes the energy domain of application of parallel plate free air ionization chambers, which to our knowledge, were used, up to now, solely for the dosimetry of hard and soft X-rays.

\section{ACKNOWLEDGMENTS}

This work was partially funded through a CNOUS France-Algeria financial support (PROFAS). The authors would like to acknowledge Dr. Wassim Ksouri and Dr. Jean-Marc Bordy from the French National Henri Becquerel Laboratory (LNHB) for their valuable comments, hints and support during the conception of the Parallel-Plate Free-Air Ionization Chamber.

\section{REFERENCES}

${ }^{1}$ D. T. Goodhead and H. Nikjoo, Radiat. Prot. Dos. 31, 343 (1990).

${ }^{2}$ C. K. Hill, B. E. Nelms, J. F. MacKay, D. W. Pearson, W. S. Kennan, T. R. Mackie, J. P. M. DeLuca, M. J. Lindstrom, and M. N. Gould, Radiat. Res. 150, 513 (1998).

${ }^{3}$ M. Gould, B. Nelms, C. Hill, J. Mackay, M. Lindstrom, T. Mackie, and P. Deluca, J. Radiat. Res. 40, 66 (1999).

${ }^{4}$ M. Hill, D. Stevens, K. S. Townsend, and D. Goodhead, Radiat. Res. 155, 503 (2001).

${ }^{5}$ M. Folkard, G. Schettino, B. Vojnovic, S. Gilchrist, A. Michette, S. Pfauntsch, K. Prise, and B. Michael, Radiat. Res. 156, 796 (2001).

${ }^{6}$ Z. Cai, P. Cloutier, D. Hunting, and L. Sanche, J. Phys. Chem. B 109, 4796 (2005). 
${ }^{7}$ P. Bernhardt, W. Friedland, P. Jacob, and H. Paretzke, Int. J. Mass. Spect. 223-224, 579 (2003).

${ }^{8}$ E. Brun, P. Cloutier, C. Sicard-Roselli, M. Fromm, and L. Sanche, J. Phys. Chem. B 113, 10008 (2009).

${ }^{9}$ K. Kobayashi, K. Hieda, H. M. znd Y. Furusawa, M. Suzuki, and T. Ito, Int. J. Radiat. Biol. 59, 643 (1991).

${ }^{10}$ M.-A. H. du Penhoat, A. Eschenbrenner, F. Abel, A. Boissiere, J.-M. Guigner, A. Chetioui, M.-F. Politis, A. Touati, E. Sage, T. Jenner, D. Stevens, and M. Hill, Int. J. Radiat. Biol. 86, 205 (2010).

${ }^{11}$ G. Neary, J. Savage, and H. Evans, Int. J. Radiat. Biol. 8, 1 (1964).

${ }^{12}$ D. Goodhead and J. Thacker, Int. J. Radiat. Biol. 31, 541 (1977).

${ }^{13}$ J. Carter, N. Cheng, Y. Qu, G. D. Suarez, and T. Guo, J. Colloid Interface Sci. 378, 70 $375 \quad(2012)$.

${ }^{14}$ J. Solomon and W. Baun, Rev. Sci. Instrum. 40, 1458 (1969).

${ }^{15}$ N. Ounoughi, C. Mavon, A. Belafrites, J.-E. Groetz, and M. Fromm, Nucl. Instr. and Meth. B 305, 61 (2013).

${ }^{16}$ A. Ferrari, A. Fassó, J. Ranft, and P. Sala, FLUKA: A Multi-Particle Transport Code (CERN-2005-10, INFN/TC05/11, SLAC-R-773, 2005).

${ }^{17}$ G. Battistoni, S. Muraro, S. Sala, F. Cerutti, A. Ferrari, S. Roesler, A. Fassò, J. Ranft, and M. Albrow., in AIP Conference Proceeding (2006) pp. 31-49.

${ }^{18}$ F. Salvat, J. Fernández-Varea, and J. Sempau, PENELOPE-2011: A code system for Monte Carlo simulation of electron and photon transport (NEA/NSC/DOC(2011)5, 2011).

${ }^{19}$ COMSOL Multiphysics User's Guide, version 4.3 (COMSOL AB, 2012).

${ }^{20}$ G. Knoll, Radiation Detection and Measurement, 4th ed. (John Wiley and Sons, 2010).

${ }^{21}$ W. Ksouri, M. Denoziere, N. Lecerf, and E. Leroy, Revue Française de Métrologie 20, 9 (2009).

${ }^{22}$ D. Burns and L. Büermann, Metrologia 46, 9 (2009).

${ }^{23}$ D. Lea, Amer. J. Roentgenol. 45, 614 (1941).

${ }^{24}$ M. Hoshi, D. Goodhead, D. Brenner, D. Bances, J. Chmielewski, M. Paciotti, and J. Bradbury, Phys. Med. Biol. 30, 1029 (1985).

${ }^{25}$ M. Folkard, B. Vojnovic, K. Prise, and B. Michael, Phys. Med. Biol. 32, 1615 (1987).

${ }^{26}$ J. Aitken, Phys. Med. Biol. 3, 27 (1958). 
${ }^{27}$ L. Büermann, B. Grosswendt, and H. Karmer, Phys. Med. Biol. 51, 5125 (2006).

${ }^{28}$ H. Buhr, L. Büermann, M. Gerlach, M. Krumrey, and H. Rabus, Phys. Med. Biol. 57, 8231 (2012).

${ }^{29}$ Constante physique pour les étalons de mesures (BIPM $\left.11 \mathrm{R} 45,1985\right)$.

${ }^{30}$ D. Burns, Consistent set of calculated values for electron-loss and photon-scatter correc400 tions for parallel-plate free-air chambers (CCRI(I)/99-4, BIPM, 1999).

${ }^{31}$ N. Ipe, K. Rosser, C. Moretti, J. Manning, and M. Palmer, Phys. Med. Biol. 46, 2107 (2001).

${ }^{32}$ P. McCaffrey, E. Mainegra-Hing, I. Kawrakow, K. Shortt, and D. Rogers, Phys. Med. Biol. 49, 2491 (2004).

${ }^{33}$ N. Takata, T. Kurosawa, A. Begumb, and T. Sugita, NIST Special Publication (2001).

${ }^{34}$ P. Lamperti and M. O'Brien, NIST Special Publication 250-58 (2001).

${ }^{35}$ J. Holt, A. Buffa, D. Perry, I. Ma, and J. McDonald, Int. J. Radiat. Onc. Biol. Phys 5, 2031 (1979).

${ }^{36}$ S. Lang, J. Hrbacek, A. Leong, and S. Klöck, Phys. Med. Biol. 57, 2819 (2012).

${ }_{410}^{37}$ J. Havercroft and S. Klevenhagen, Phys. Med. Biol 38, 25 (1993).

${ }^{38}$ J. Burns and D. Burns, Phys. Med. Biol 38, 1986 (1993).

${ }^{39}$ D. Rogers, I. Kawrakow, N. Klassen, J. McCaffrey, C. Ross, K. Shortt, L. van der Zwan, and G. Daskalov, 1999-2001 Report to CCRI(I) Meeting (BIPM, 2001).

${ }^{40}$ F. Botta, A. Mairani, G. Battistoni, M. Cremonesi, A. D. Dia, A. Fasso, A. Ferrari,

${ }^{41}$ C. Champion, S. Incerti, Y. Perrot, R. Delorme, M. Bordage, M. Bardies, B. Mascialino, H. Tran, V. Ivanchenko, M. Bernal, Z. Francis, J.-E. Groetz, M. Fromm, and L. Campos, Appl. Radiat. Isot. 83, 137 (2014).

${ }^{42}$ M. Berger, J. Coursey, M. Zucker, and J. Chang, (2005).

${ }^{43}$ O. Boulanouar, A. Khatyr, G. Herlem, F. Palmino, L. Sanche, and M. Fromm, J. Phys. Chem. C 115, 21291 (2011). 